Revisão / Review

\title{
Deficiência de ferro no idoso
}

\section{Iron deficiency in the elderly}

Sandra F. M. Gualandro

Naira H. S. L. Hojaij ${ }^{2}$

Wilson Jacob Filho ${ }^{3}$

\begin{abstract}
Anemia é comum em idosos e é associada a significante morbidade e mortalidade. Mais de 10\% dos indivíduos acima de 65 anos tem anemia. Com uma proporção crescente da população mundial atingindo idade igual ou superior a 65 anos, a prevalência de anemia certamente aumentará no futuro. $O$ diagnóstico precoce é importante para prevenir piora do quadro, diminuir progressão da doença e melhorar a evolução dos pacientes. Os critérios mais utilizados em estudos epidemiológicos para definir anemia em idosos são os da OMS (hemoglobina $<12 \mathrm{~g} / \mathrm{dL}$ para mulheres e hemoglobina $<13 \mathrm{~g} / \mathrm{dL}$ para homens). Aproximadamente um terço dos idosos com anemia tem deficiência de ferro, folato elou vitamina B12, um terço tem insuficiência renal elou inflamação crônica e o terço remanescente tem anemia inexplicada. A anemia ferropênica é microcítica e hipocrômica e caracteriza-se por baixos níveis de ferritina sérica, capacidade total de ligação de ferro do plasma aumentada, saturação da transferrina diminuída, concentração do receptor solúvel da transferrina elevada e ausência de ferro na medula óssea. É causada geralmente por perda de sangue pelo trato gastrointestinal devido a gastrite, úlceras, câncer de colo ou angiodisplasia. Anormalidades do trato gastrointestinal podem ser identificadas na maioria dos pacientes. Em alguns casos, ingestão ou absorção inadequada de ferro pode contribuir para a anemia. Entretanto, em todos os casos deveria ser exaustivamente investigada e excluida perda de sangue antes de assumir que a deficiência de ferro é devida a outras causas. O tratamento inclui parar o sangramento e repor o ferro. Rev. Bras. Hematol. Hemoter. 2010; 32(Supl.2):57-61.
\end{abstract}

Palavras-chave: Deficiência de ferro; anemia; idoso.

\section{Introdução}

O envelhecimento populacional, que ocorre de maneira vertiginosa desde o início do século XX nos países desenvolvidos e a partir dos anos 60 no Brasil, traz um dos maiores desafios para a saúde pública contemporânea. No Brasil, no período de 1960 a 2002, o número de indivíduos com mais de 60 anos aumentou em $500 \%$, passando de 3 para 14 milhões e estima-se que alcançará 32 milhões em 2020.
Dois fenômenos epidemiológicos foram determinantes para esta transição demográfica: a redução da mortalidade documentada desde o censo de 1940 e, principalmente, o declínio progressivo da fecundidade na segunda metade do século passado. Este rápido e progressivo aumento do contingente de adultos e idosos foi o responsável pela mudança no perfil de doenças no mundo, ou transição epidemiológica, predominando as condições crônicas de acometimento da saúde. O Brasil, assim como os demais países em

${ }^{1}$ Hematologista. Professora da Disciplina de Hematologia e Hemoterapia da Faculdade de Medicina da Universidade de São Paulo

-FMUSP - São Paulo-SP.

${ }^{2}$ Geriatra. Médica Assistente do Serviço de Geriatria do Hospital das Clínicas da Faculdade de Medicina da Universidade de São Paulo HC-FMUSP - São Paulo-SP.

${ }^{3}$ Geriatra. Professor Titular da Disciplina de Geriatria da Faculdade de Medicina da Universidade de São Paulo - FMUSP - São Paulo-SP.

Faculdade de Medicina da Universidade de São Paulo; Hospital das Clínicas da Faculdade de Medicina da Universidade de São Paulo São Paulo-SP.

Correspondência: Sandra Fátima Menosi Gualandro

Rua Dr. Eneas de Carvalho Aguiar, 155 - $1^{\circ}$ andar - Cerqueira Cesar

05403-000 - São Paulo-SP - Brasil

E-mail: sandrafmg@uol.com.br

Doi: 10.1590/S1516-84842010005000058 
desenvolvimento, passa por uma transição prolongada, já que ainda persistem taxas elevadas de doenças infecto-parasitárias, ao lado das condições crônicas em ascensão. ${ }^{1}$

A ocorrência simultânea de múltiplas doenças ou condições médicas num mesmo indivíduo, definida por van den Akken em 1962 como multimorbidade, foi o primeiro grande impacto da transição demográfica e epidemiológica acima descritas. De maneira geral, a presença de pelo menos uma condição crônica ocorre em $45 \%$ da população e em mais de $80 \%$ dos idosos, e metade de todos estes indivíduos apresenta condições crônicas múltiplas. No Brasil, o primeiro estudo epidemiológico longitudinal com idosos residentes na comunidade foi o projeto Epidoso, no qual foram acompanhados, por dois anos (1991 e 1992), 1.667 indivíduos com 65 anos ou mais, residentes na cidade de São Paulo. Neste estudo, apenas $6 \%$ dos idosos consideravam-se livres de doenças e $33,2 \%$ relatavam pelo menos cinco condições crônicas. ${ }^{3}$

Mais recentemente, o projeto multicêntrico SABE (Saúde, Bem-Estar e Envelhecimento), patrocinado e coordenado pela Organização Pan-Americana de Saúde e implementado no Brasil por pesquisadores da Faculdade de Saúde Pública da Universidade de São Paulo, mostrou, após entrevistar 2.143 idosos residentes na área urbana de São Paulo, que, além da grande prevalência autorreferida de doenças crônicas, como hipertensão arterial e artrite/artrose/reumatismo, existem outras condições igualmente preocupantes nos idosos. ${ }^{4}$

Provavelmente. o maior impacto das condições crônicas na saúde do idoso seja o prejuízo funcional para a realização de atividades cotidianas de vida. Uma das maneiras de medir este impacto é através de estudos de carga de doença, que conseguem trazer outra dimensão que não somente a existência ou não de doenças, mas, também, o impacto nos anos de vida perdidos por isso. Segundo o Estudo da Carga de Doença do Brasil, as doenças crônico-degenerativas responderam por $66,3 \%$ da carga de doença, as doenças infecciosas por $23,5 \%$ e as causas externas por $10,2 \%$, uma vez mais comprovando a transição epidemiológica no Brasil. ${ }^{5}$ A saúde, portanto, já não pode mais ser medida pela presença ou não de doenças, mas sim pelo grau de preservação da capacidade funcional, determinando então um envelhecimento bem ou mal sucedido.

Dados demográficos e estudos recentes indicam que anemia é uma ocorrência frequente na população idosa e que sua prevalência aumenta com a idade. Embora a anemia possa refletir uma doença de base, por vezes não diagnosticada, ela é também um fator de risco independente para morbidade e mortalidade. Além disso, interfere no desempenho físico e mental e na habilidade para manter as atividades do cotidiano, afetando a qualidade de vida. ${ }^{6}$

Dentro desse panorama, é de fundamental importância o estudo de processos potencialmente reversíveis, como as deficiências nutricionais e, entre elas, a deficiência de ferro, para que possamos minimizar a evolução para a incapacidade e morte.

\section{Definição de anemia no idoso e epidemiologia}

A anemia na população geral tem, tradicionalmente, sido definida de acordo com os critérios estabelecidos pela Organização Mundial de Saúde (OMS): hemoglobina $<13,0 \mathrm{~g} / \mathrm{dL}$ em homens e $<12 \mathrm{~g} / \mathrm{dL}$ em mulheres.

Embora vários especialistas sugiram a substituição dos padrões da OMS por definições baseadas em estudos mais recentes em idosos, por enquanto critérios revisados não têm sido adotados, e os critérios da OMS permanecem amplamente utilizados. Um estudo longitudinal de dez anos em residentes da comunidade na Holanda concluiu que os critérios da OMS são apropriados para esta população.?

A anemia é uma condição comum no idoso, geralmente multifatorial e, como já citado, sua prevalência aumenta com a idade. Embora o declínio da hemoglobina tenha sido, no passado, considerado uma consequência normal do envelhecimento, as evidências acumuladas demonstram que a presença de anemia nas pessoas idosas reflete saúde comprometida e aumento da vulnerabilidade para desfechos adversos. ${ }^{8}$ Mais de $10 \%$ dos adultos residentes na comunidade com idade igual ou superior a 65 anos têm anemia, de acordo com os critérios da OMS. Após os 50 anos de idade, a prevalência de anemia aumenta à medida que a idade avança e excede $20 \%$ naqueles com 85 anos ou mais. Entre os idosos institucionalizados, a anemia está presente em $48 \%$ a $63 \%$ dos residentes. ${ }^{9}$

As causas de anemia têm sido divididas em três grandes grupos: um terço com deficiência nutricional, um terço com anemia das doenças crônicas, atualmente chamada de anemia da inflamação crônica e/ou insuficiência renal crônica, e o terço restante com anemia inexplicada. ${ }^{8,10,11}$ A deficiência de ferro sozinha é responsável por quase metade dos casos de anemias causadas por deficiências nutricionais. ${ }^{10} \mathrm{Na}$ Europa e nos EUA, anemia ferropênica ocorre em aproximadamente $4 \%$ a $5 \%$ dos idosos. ${ }^{12}$

\section{Etiologia}

A deficiência de ferro é comum no idoso e resulta de perda crônica de sangue causada geralmente por gastrite droga-induzida pelo uso de anti-inflamatórios não esteroides, úlceras gastrointestinais, câncer de colo, divertículos ou angiodisplasia. ${ }^{11-13} \mathrm{Em}$ alguns casos, dieta inadequada ou absorção inadequada de ferro podem contribuir para a anemia, mas perda de sangue deve ser exaustivamente investigada e excluída antes de assumir que a deficiência de ferro decorre de outras causas. ${ }^{13}$ Anormalidades do trato gastrointestinal podem ser identificadas na maioria dos idosos com anemia ferropênica. ${ }^{10,11}$ Endoscopia gastrointestinal realizada em cem pacientes idosos consecutivos com anemia ferropênica mos- 
trou que $62 \%$ tinham uma lesão que poderia causar perda de sangue e $16 \%$ tinham pólipos pré-malignos ou câncer de colon. ${ }^{10}$ Dentre as causas gastrointestinais que levam à carência de ferro, uma que tem sido subestimada é a doença celíaca. A doença celíaca tem sido diagnosticada tradicionalmente em crianças e adultos jovens, mas, nos últimos anos, tem aumentado a sua detecção na população idosa. Existe uma conscientização crescente de que ela pode ocorrer no idoso, embora as apresentações com diarreia, perda de peso ou ambas sejam menos comuns e isto leve a grandes atrasos no diagnóstico. ${ }^{14}$

\section{Repercussões clínicas}

Embora a maioria dos casos de anemia em idosos seja leve, seu impacto sobre a morbidade, mortalidade e custos para o sistema de saúde é significante. Vários estudos têm implicado a anemia como um fator de risco independente para uma variedade de condições clínicas e de desfechos adversos tanto em residentes da comunidade como em indivíduos institucionalizados. ${ }^{6,15}$

O idoso, pela natural redução de suas reservas fisiológicas, sofre mais rapidamente as consequências da anemia, principalmente nas perdas sanguíneas agudas, pela menor capacidade de adaptação cardiovascular e respiratória. Além disso, os sinais clássicos de palidez cutâneo-mucosa, taquicardia e dispneia poderão ser mascarados pelas próprias características da senescência, por outras comorbidades ou pelo uso de alguns medicamentos; como exemplo, os betabloqueadores. A morbidade da anemia é maior nesta população, com maior prevalência de complicações, como infarto do miocárdio, acidente vascular cerebral, insuficiência arterial periférica, isquemia mesentérica, sendo necessária, portanto, atenção especial nos casos de anemia de instalação rápida.

$\mathrm{O}$ risco de mortalidade é significativamente mais alto entre idosos anêmicos que nos não anêmicos. Esta associação foi observada em vários estudos de grandes coortes e permanece significante mesmo após a exclusão dos indivíduos com comorbidades. ${ }^{9}$ O impacto sobre a mortalidade naqueles com comorbidades é grande. ${ }^{7,16,17}$ Exemplos desta relação têm sido mostrados em idosos com insuficiência cardíaca comparados com coortes semelhantes de não anêmicos ${ }^{18} \mathrm{e}$ em indivíduos que apresentaram melhora em insuficiências orgânicas associada à melhora nos níveis de hemoglobina. ${ }^{19}$

\section{Abordagem diagnóstica}

Como a anemia é sempre secundária a alguma doença de base, outros sintomas, além dos relacionados à redução do transporte de oxigênio, podem ocorrer, sendo sempre muito importantes a anamnese detalhada, incluindo informações sobre a presença de comorbidades e sobre o uso de medicamentos como anti-inflamatórios não hormonais, anticoa- gulantes, diuréticos (podem mascarar a presença de anemia) e de álcool, assim como o exame físico minucioso.

Da mesma maneira, para qualquer paciente com suspeita de anemia é importante a realização de hemograma completo com contagem de reticulócitos. A anemia decorrente de carência de ferro é tipicamente microcítica e hipocrômica, com reticulócitos baixos, caracterizando uma anemia por falta de produção.

Níveis baixos de ferritina sérica, capacidade total de ligação de ferro do plasma elevada, saturação da transferrina diminuída, concentração de receptor solúvel de transferrina (sTfr) elevada e ausência de estoques de ferro na medula óssea acompanham a anemia por deficiência de ferro. ${ }^{13}$

A alta prevalência da deficiência de ferro entre as causas de anemia no idoso enfatiza a importância do reconhecimento deste diagnóstico. Ao mesmo tempo revela como os atuais métodos diagnósticos podem ser pouco acurados para este grupo de pacientes. Isto ocorre devido à concomitante presença de doenças crônicas nesta população. A determinação dos estoques de ferro da medula óssea é o gold standard para o diagnóstico acurado, mas é claramente impraticável para o propósito de screening. A medida do sTfr e da ferritina sérica poderia fornecer o grau adequado de acurácia diagnóstica. ${ }^{20}$ No entanto, apesar dos níveis de sTfr expressos como uma relação do $\log$ da ferritina sérica (índice de sTfr) terem sido reportados como a ferramenta preditiva mais útil para acessar os estoques de ferro, a dosagem do sTfr não é uniformemente disponível nem amplamente padronizada em laboratórios clínicos. ${ }^{11} \mathrm{~A}$ ferritina sérica, por sua vez, se eleva em presença de inflamação, situação frequente nesta população. Dessa maneira, a associação entre anemia por deficiência de ferro e anemia das doenças crônicas (ou anemia da inflamação crônica) pode dificultar a identificação da ferropenia, especialmente nos estágios iniciais. ${ }^{10}$

Após o diagnóstico de deficiência de ferro ser feito, é muito importante pesquisar as causas, começando pela busca exaustiva de perdas de sangue, especialmente pelo tubo digestivo. $\mathrm{O}$ avanço da idade tem sido identificado como preditor de afecções gastrointestinais. A investigação de escolha é a endoscopia do trato gastrointestinal superior e inferior, pela sua acurácia, taxa de complicações aceitável e potencial para intervenções terapêuticas sem aumento da mortalidade precoce. ${ }^{12} \mathrm{~A}$ investigação deve ser instituída imediatamente após a deteç̧ão da deficiência de ferro. Em um estudo sobre oportunidades perdidas para avaliação endoscópica para diagnóstico de câncer colorretal, a anemia foi a pista associada com o tempo mais longo para encaminhamento à endoscopia. ${ }^{21}$ Sangramentos gastrointestinais obscuros do intestino delgado podem não ser visualizados na endoscopia digestiva alta ou na colonoscopia. Nos casos de endoscopia digestiva alta e colonoscopia aparentemente normais, pode-se tentar localizar as perdas através da utilização de métodos radioisotópicos ou de cápsula endoscópica. 
A cápsula endoscópica, um método de utilização relativamente recente, possibilita a visualização de toda a mucosa do intestino delgado e desempenha um papel crítico para a avaliação dos pacientes com endoscopia digestiva alta e colonoscopia negativas. ${ }^{22}$

\section{Prevenção}

Com o aumento da população idosa, um aumento na incidência das condições de saúde relacionadas à idade é esperado. Destas, a anemia merece interesse especial porque é potencialmente reversível com tratamento apropriado, o qual pode também retardar ou prevenir morbidades relacionadas ao envelhecimento. Portanto, apesar da alta prevalência, ela não deve ser considerada como consequência inevitável do envelhecimento.

A inclusão do hemograma completo nos exames de rotina da população idosa permitiria a detecção precoce de anemia, e a análise dos índices hematológicos e do número de reticulócitos possibilitaria a instituição da investigação apropriada, considerando que, como já citado, ela é fator de risco independente para morbidade e mortalidade. ${ }^{6}$

No caso da anemia por deficiência de ferro, a principal causa é representada por sangramento pelo trato gastrointestinal, sendo bem menos frequentes a ingestão e a absorção inadequadas. A reposição do ferro deve ser encarada como tratamento da repercussão da doença de base. É importante identificar a causa e iniciar o tratamento adequado para prevenir o agravamento da anemia, diminuir a progressão da doença e melhorar o prognóstico dos pacientes. ${ }^{13}$

\section{Abstract}

Anemia is a common problem in the elderly and is associated with significant morbidity and mortality. More than $10 \%$ of all individuals above the age of 65 have anemia. Because an increasing proportion of the world's population is aged 65 and older, it is inevitable that the prevalence of anemia will increase in the future. Thus, early diagnosis of anemia is important to prevent the condition from worsening, to slow disease progression, and improve outcomes in patients. The WHO definition of anemia (hemoglobin concentration $<12 \mathrm{~g} / \mathrm{dL}$, in women and $<13 \mathrm{~g} / \mathrm{dL}$, in men) is most often used in epidemiologic studies of older adults. Among older adults with anemia approximately one-third have evidence of iron, folate, and/or vitamin B12 deficiency, another third have renal insufficiency and/or chronic inflammation, and the remaining third have anemia that is unexplained. Anemia due to iron deficiency (IDA) is microcytic and hypochromic. Low serum ferritin levels, high total iron-binding capacity, low transferrin saturation, high concentrations of soluble transferrin receptor, and absent bone marrow iron stores accompany IDA. Iron deficiency in the elderly usually occurs as a result of chronic gastrointestinal blood loss caused by gastritis, ulcers, colon cancer, or angiodysplasia. Gastrointestinal tract abnormalities can be identified in the majority of patients with IDA. In some cases, inadequate intake or inadequate absorption of iron may contribute to the anemia. However, in all cases blood loss should be investigated and excluded before assuming that iron deficiency is due to other causes. Treatment includes stopping blood loss and replacing iron. Rev. Bras. Hematol. Hemoter. 2010;32(Supl.2):57-61.

Key words: Iron deficiency; anemia; elderly.

\section{Referências Bibliográficas}

1. Carvalho JAM, Garcia RA. O envelhecimento da população brasileira: um enfoque demográfico. Cad Saúde Pública. 2003; 19:725-33.

2. van den Akker M, Buntinx F, Roos S, Knottnerus JA. Comorbidity or multimorbidity: what's in a name? A review of the literature. Eur J Gen Pract 1996;2(2):65-70.

3. Ramos LR. Fatores determinantes do envelhecimento saudável em idosos residentes em centro urbano: Projeto Epidoso, São Paulo. Cad Saúde Pública 2003;19(3):793-8.

4. Lebrão ML, Laurenti R. Saúde, bem-estar e envelhecimento: o estudo SABE no Município de São Paulo. Rev Bras Epidemiol. $2005 ; 8(2): 127-41$.

5. Schramm JMA, Oliveira AF, Leite IC, Valente JG, Gadelha AMJ, Portela MC, et al. Transição epidemiológica e o estudo de carga de doença no Brasil. Ciênc Saúde Coletiva. 2004;9:897-908.

6. Laudicina RJ. Anemia in an aging population. Clin Lab Sei. 2008; 21(4):232-9.

7. Izaks GJ, Westendorf RG, Knook DL. The definition of anemia in older persons. JAMA. 1999;281(18):1714-7.

8. Guralnik JM, Eisenstaedt RS, Ferrucci L, Klein HG, Woodman RC. Prevalence of anemia in persons 65 years and older in the United States: evidence for a high rate of unexplained anemia. Blood. 2004;104(8):2263-8.

9. Patel KV, Guralnik JM. Prognostic implications of anemia in older adults. Haematologica. 2009;94(1):1-2.

10. Patel KV. Epidemiology of anemia in older adults. Semin Hematol. 2008;45(4):210-7.

11. Eisenstaedt R, Penninx BW, Woodman RC. Anemia in the elderly: current understanding and emerging concepts. Blood Rev. 2006; 20(4):213-26.

12. Nahon S, Lahmek P, Aras N, Poupardin C, Lesgourgues B, Macaigne $\mathrm{G}$, et al. Management and predictors of early mortality in elderly patients with iron deficiency anemia: a prospective study of 111 patients. Gastroenterol Clin Biol. 2007;31(2):169-74.

13. Balducci L. Epidemiology of anemia in the elderly: information on diagnostic evaluation. J Am Geriatr Soc. 2003;51(3 Suppl.):S2-S9.

14. Freeman HJ. Adult celiac disease in the elderly. World J Gastroenterol. 2008;14(45):6911-4.

15. Lipschitz D. Medical and functional consequences of anemia in the elderly. J Am Geriatr Soc. 2003;51(3 Suppl):S10-S3.

16. Penninx BW, Pahor M, Woodman RC, Guralnik JM. Anemia in old age is associated with increased mortality and hospitalization. J Gerontol A Biol Sci Med Sci. 2006;61(5):474-9.

17. Denny SD, Kuchibhatla MN, Cohen HJ. Impact of anemia on mortality, cognition, and function in community-dwelling elderly. Am J Med. 2006;119(4):327-34.

18. Ezekowitz JA, McAlister FA, Armstrong PW. Anemia is common in heart failure and is associated with poor outcomes: insights from a cohort of 12.065 patients with new-onset heart failure. Circulation. 2003;107(2):223-5. 
19. Hayashi T, Suzuki A, Shoji T, Togawa M, Okada N, et al. Cardiovascular effect of normalizing the hematocrit level during erythropoietin therapy in predialysis patients with chronic renal failure. Am J Kidney Dis. 2000;35(2):250-6.

20. Guralnik JM, Ershler WB, Schrier SL, Picozzi VJ. Anemia in the elderly: a public health crisis in hematology. Hematology Am Soc Hematol Educ Program. 2005:528-32.

21. Singh H, Daci K, Petersen LA, Collins C, Petersen NJ, et al. Missed opportunities to initiate endoscopic evaluation for colorectal cancer diagnosis. Am J Gastroenterol. 2009;104(10):2543-54.

22. Muhammad A, Pitchumoni CS. Evaluation of iron deficiency anemia in older adults: the role of wireless capsule endoscopy. J Clin Gastroenterol. 2009;43(7):627-31.

O tema foi sugerido e avaliado pelo coeditor deste fascículo educativo, Rodolfo Delfini Cançado, e pelo board interno da RBHH, e publicado após a concordância do editor, Milton Artur Ruiz.

Conflito de interesse: sem conflito de interesse

Recebido: 22/12/2009

Aceito: 16/01/2010 